\title{
Epigenetic DNA changes in childhood acute lymphoblastic leukemia - the drug treatment effect.
}

\section{Rafal Rozalski ( $\nabla$ rafalr@cm.umk.pl )}

Department of Clinical Biochemistry, Faculty of Pharmacy, Collegium Medicum in Bydgoszcz, Nicolaus Copernicus University in Toruń

\section{Daniel Gackowski}

Department of Clinical Biochemistry, Faculty of Pharmacy, Collegium Medicum in Bydgoszcz, Nicolaus Copernicus University in Toruń

\section{Aleksandra Skalska}

Department of Clinical Biochemistry, Faculty of Pharmacy, Collegium Medicum in Bydgoszcz, Nicolaus Copernicus University in Toruń

\section{Marta Starczak}

Department of Clinical Biochemistry, Faculty of Pharmacy, Collegium Medicum in Bydgoszcz, Nicolaus Copernicus University in Toruń

\section{Agnieszka Siomek-Gorecka Siomek-Gorecka}

Department of Clinical Biochemistry, Faculty of Pharmacy, Collegium Medicum in Bydgoszcz, Nicolaus Copernicus University in Toruń

\section{Ewelina Zarakowska}

Department of Clinical Biochemistry, Faculty of Pharmacy, Collegium Medicum in Bydgoszcz, Nicolaus Copernicus University in Toruń

\section{Martyna Modrzejewska}

Department of Clinical Biochemistry, Faculty of Pharmacy, Collegium Medicum in Bydgoszcz, Nicolaus Copernicus University in Toruń

\section{Tomasz Dziaman}

Department of Clinical Biochemistry, Faculty of Pharmacy, Collegium Medicum in Bydgoszcz, Nicolaus Copernicus University in Toruń

\section{Anna Szpila}

Department of Clinical Biochemistry, Faculty of Pharmacy, Collegium Medicum in Bydgoszcz, Nicolaus Copernicus University in Toruń

\section{Kinga Linowiecka}

Department of Human, Biology, Institute of Biology, Faculty of Biological and Veterinary Sciences, Nicolaus Copernicus University in Toruń

Jolanta Guz

Department of Clinical Biochemistry, Faculty of Pharmacy, Collegium Medicum in Bydgoszcz, Nicolaus Copernicus University in Toruń 


\section{Justyna Szpotan}

Department of Human, Biology, Institute of Biology, Faculty of Biological and Veterinary Sciences, Nicolaus Copernicus University in Toruń

\section{Maciej Gawronski}

Department of Clinical Biochemistry, Faculty of Pharmacy, Collegium Medicum in Bydgoszcz, Nicolaus Copernicus University in Toruń

\section{Anna Labejszo}

Department of Geriatrics, Division of Biochemistry and Biogerontology, Collegium Medicum in Bydgoszcz, Nicolaus Copernicus University in Toruń

\section{Lidia Gackowska}

Department of Immunology, Faculty of Pharmacy, Collegium Medicum in Bydgoszcz, Nicolaus Copernicus University in Toruń

\section{Marek Foksinski}

Department of Clinical Biochemistry, Faculty of Pharmacy, Collegium Medicum in Bydgoszcz, Nicolaus Copernicus University in Toruń

\section{Elwira Olinska}

District Health Center in Kartuzy

\section{Aleksandra Wasilow}

Department of Clinical Biochemistry, Faculty of Pharmacy, Collegium Medicum in Bydgoszcz, Nicolaus Copernicus University in Toruń

\section{Andrzej Koltan}

Department of Pediatric Hematology and Oncology, Collegium Medicum, Nicolaus Copernicus University in Toruń

\section{Jan Styczynski}

Department of Pediatric Hematology and Oncology, Collegium Medicum, Nicolaus Copernicus University in Toruń

\section{Ryszard Olinski}

Department of Clinical Biochemistry, Faculty of Pharmacy, Collegium Medicum in Bydgoszcz, Nicolaus Copernicus University in Toruń

\section{Research Article}

Keywords: DNA demethylation, pediatric cancer, childhood acute lymphoblastic leukemia (ALL)

Posted Date: April 26th, 2021

DOI: https://doi.org/10.21203/rs.3.rs-427067/v1

License: (c) (1) This work is licensed under a Creative Commons Attribution 4.0 International License. Read Full License 


\section{Abstract}

The active DNA demethylation process may be linked with aberrant methylation and may be involved in leukemogenesis. We investigated the role of epigenetic DNA modifications in childhood acute lymphoblastic leukemia (ALL) development. We analyzed the levels of 5-methyl-2'-deoxycytidine (5-mdC) oxidation products in the cellular DNA and urine of children with ALL (at diagnosis and after completion of chemotherapy, $n=55$ ) using two-dimensional ultra-performance liquid chromatography with tandem mass spectrometry (2D UPLC-MS/MS). Moreover, the expression of Ten Eleven Translocation enzymes (TETs) at the mRNA and protein levels was determined. Additionally, the ascorbate level in blood plasma was analyzed. Before treatment, the ALL patients had profoundly higher levels of the analyzed modified DNA in their urine than the controls. After chemotherapy, we observed a statistically significant decrease in active demethylation products in urine, with the final level similar to the level characteristic of healthy children. The level of 5-hmdC on the DNA of the blood leukocytes in the patient group was significantly lower than it was in the control group. Our data suggest that urinary excretion of epigenetic DNA modification may be a marker of pediatric ALL pathogenesis and a reliable marker of chemotherapy response.

\section{Introduction}

Acute lymphoblastic leukemia (ALL) is the most common pediatric malignancy. ALL can develop from any lymphoid cell blocked at a particular stage of development and usually originates in a single B-or Tlymphocyte progenitor ${ }^{1}$. Most patients diagnosed with ALL have genetic aberrations that contribute to a higher rate of proliferation and impaired differentiation of lymphoid hematopoietic progenitors ${ }^{2}$.

One of the reasons for the observed genetic alterations may be aberrant DNA methylation that leads to changes in the expression of hematopoietic genes. Moreover, in several studies, it has been observed that the cytosine methylation pattern of leukemic cells differ from that of nonleukemic cells ${ }^{3-5}$.

DNA methylation has been studied as a stable epigenetic modification for decades. However, despite its stability, DNA methylation may be reversed via an active DNA demethylation process, as described recently (Figure 1). This demethylation process involves enzymatic oxidation (with Ten Eleven Translocation enzymes - TET enzymes) with the subsequent formation of 5-hydroxymethylcytosine (5hmCyt), which can be further oxidized to 5-formylcytosine (5-fCyt) and 5-carboxylcytosine (5-caCyt) ${ }^{6,7}$. Both 5-fCyt and 5-caCyt are subsequently removed by base excision repair (BER) or nucleotide excision repair (NER) and replaced by cytosine to complete the process of active DNA demethylation ${ }^{8}$. 5Hydroxymethyluracil (5-hmUra) may also be generated by TET enzymes and removed via the BER pathway. The eliminated bases (or deoxynucleosides) are released into the bloodstream and eventually are also found in urine ${ }^{9}$.

It is likely that active DNA demethylation is linked with aberrant methylation and may be involved in leukemogenesis. Indeed, hematological malignancies were among the first in which aberrant 
demethylation status was discovered, and the TET gene was initially defined as a fusion partner of the mixed lineage leukemia (MLL) gene in acute myeloid leukemia (AML). Importantly, TET inactivation leads to the dramatic deregulation of hematopoiesis that, in turn, triggers blood malignancies ${ }^{10}$.

Quantification of epigenetic DNA modifications may elucidate the consequences of TET expression deficiency. The functional consequences of epigenetic changes may be critical for disease initiation and progression. However, changes in the level of epigenetic DNA modifications may also mirror environmental changes linked with drug delivery.

We thus sought to determine an array of endogenously generated DNA base modifications (5-hmUra, 5mCyt, 5-hmCyt, 5-fCyt, 5-caCyt), with the goal of determining whether these modifications can serve as predictive biomarkers of leukemia disease process. TET enzyme expression at the mRNA and protein levels was also studied. Since ascorbate may substantially enhance the generation of 5-hmCyt (as well as other DNA epigenetic modifications), probably by acting as a cofactor of TETs during the hydroxylation of 5-mCyt, its level was analyzed in the blood of the experimental groups.

Oxidative stress and impaired repair of oxidatively modified DNA have been documented for children with ALL 11,12. Therefore, we also analyzed the 8-oxo-7,8-dihydro-2'-deoxyguanosine (8-oxodG) level (an established biomarker of oxidative stress/DNA damage) in the cellular DNA and urine of the ALL patients and the control group participants.

\section{Results}

\section{Levels of epigenetic modifications and 8-oxoGua in urine and DNA}

The ALL patients before treatment had substantially higher levels of analyzed modifications in their urine than the controls, and the differences were significant for all compounds (Table 1). After chemotherapy, the patients exhibited a statistically significant decrease in active demethylation products in their urine (5hmCyt, 5-hmdC, 5-mdCyt, 5-caCyt, 5-hmUra) both 33 days after starting the treatment (point B) and six months after the start of therapy (point $C$ ). Notably, the levels of analyzed compounds at point $C$ were similar to those observed in the control group (Figure 2). Significantly elevated levels of 8-oxodG were noted in the urine of patients after chemotherapy $(2.8 \mathrm{nmol} / \mathrm{mmol}$ creatinine at point $A$ and 5.7 and 4.2 at points $B$ and C). The level of 5-hmdC in the DNA of blood leukocytes in the patient group was significantly lower than that in the control group (median value: 0.065 vs. $0.078, p=0.007$ ). An inverse relationship was observed in the case of 8-oxodG, for which the ALL patients showed significantly higher levels than the controls (1.59 vs. 1.38 , respectively, $p=0.009$, Table 2$)$. Description of initial diagnosis, based on immunophenotypes and genetic aberrations was placed in Supplementary Materials (Table S1). 


\begin{tabular}{cccc}
\hline & $\begin{array}{c}\text { Patients } \\
\text { [nmol/mmol creatinine] }]\end{array}$ & $\begin{array}{c}\text { Controls } \\
{[\mathrm{nmol} / \mathrm{mmol} \text { creatinine }]}\end{array}$ & P value \\
\hline 5-hmdC & $26.3(7.6-61.9)$ & $5.1(3.6-8.0)$ & $0.000001^{*}$ \\
8-oxodG & $2.5(1.6-4.0)$ & $1.4(1.0-1.9)$ & $0.0001^{*}$ \\
5-hmCyt & $13.6(8.7-21.8)$ & $5.5(4.5-6.9)$ & $0.000001^{*}$ \\
5-mdC & $9.1(2.2-22.7)$ & $2.2(0.7-4.3)$ & $0.00003^{*}$ \\
5-caCyt & $8.7(6.7-15.1)$ & $4.0(3.0-4.7)$ & $0.00001^{*}$ \\
5-fCyt & $3.2(2.2-4.4)$ & $2.3(1.6-3.1)$ & $0.01^{*}$ \\
5-hmUra & $30.4(22.2-55.2)$ & $13.8(10.7-18.6)$ & $0.0001^{*}$ \\
\hline
\end{tabular}

Table 1. Urinary levels of DNA damage markers and active demethylation products of 5-methylcytosine. The results are presented as median values with interquartile ranges $(* \mathrm{p}<0.05)$.

The ALL patients were divided based on the subtype of disease (B and T type) and all the analyzed parameters in urine were determined separately for this subtypes (Table S2 in Supplementary Materials). The ETV6/RUNX1 gene fusion generated by $\mathrm{t}(12 ; 21)(\mathrm{p} 13 ; \mathrm{q} 22)$, is the most frequent chromosomal lesion in children with B-ALL ${ }^{13-15}$. The ETV6/RUNX1 fusion gene was detected in 15 patients (28\%). They had substantially higher levels of analyzed modifications in urine than the ETV6/RUNX1-negative ALL patients (except 8-oxodG), and the differences were significant for 5-hmdC, 5-hmCyt, 5-fCyt and 5-hmUra (Table S3 in Supplementary Materials). Notable, after completion of chemotherapy those unprecedented high values dropped to these observed in the healthy children/control group (see Table S4 in supplementary materials). However, in the case of the DNA, no statistically significant differences were found.

\begin{tabular}{cccc}
\hline & Patients (before treatment) & Controls & P value \\
\hline $5-\mathrm{metdC} / 10 \mathrm{e}^{3} \mathrm{dN}$ & $8.7(8.6-9.0)$ & $8.8(8.6-9.0)$ & 0.41 \\
$5-\mathrm{hmdC} / 10 \mathrm{e}^{3} \mathrm{dN}$ & $0.065(0.04-0.08)$ & $0.078(0.07-0.08)$ & $0.007^{*}$ \\
\hline $5-\mathrm{fdC} / 10 \mathrm{e}^{6} \mathrm{dN}$ & $0.15(0.1-0.23)$ & $0.15(0.13-0.22)$ & 0.55 \\
\hline $5-\mathrm{cadC} / 10 \mathrm{e}^{9} \mathrm{dN}$ & nd & nd & - \\
\hline $\mathrm{dU} / 10 \mathrm{e}^{6} \mathrm{dN}$ & $5.5(3.3-6.3)$ & $4.9(4.5-5.3)$ & 0.72 \\
\hline $5-\mathrm{hmdU} / 10 \mathrm{e}^{6} \mathrm{dN}$ & $0.47(0.31-0.63)$ & $0.34(0.23-0.59)$ & 0.28 \\
\hline 8-oxodG $/ 10 \mathrm{e}^{6} \mathrm{dN}$ & $1.59(1.2-3.2)$ & $1.38(1.2-1.6)$ & $0.009^{*}$ \\
\hline
\end{tabular}

Table 2. The levels of active demethylation products of 5-methylcytosine and 8-oxodG in leukocyte DNA. The results are presented as median values with interquartile ranges $(* \mathrm{p}<0.05)$.

\section{Expression of TET and TDG mRNA}

The expression of TET1 in the ALL patients was significantly higher than that in the control. The expression of TET2 and TET3 in the individuals with ALL was significantly lower than that in the controls $(p=0.005$ and $p=0.00004$, respectively). A similar statistically significant difference was found for the expression of TDG in the ALL group and the control group ( $p=0.00001$, Table 3$)$. 


\begin{tabular}{cccc}
\hline Gene & ALL & Control & ALL 1 vs Control \\
& [mRNA expression ratio] & $\begin{array}{c}\text { P value } \\
\text { [mRNA expression ratio] }\end{array}$ & $0.013^{*}$ \\
\hline TET1 & 0.002 & 0.001 & \\
& $(0.001-0.012)$ & $(0.0007-0.0015)$ & $0.005^{*}$ \\
TET2 & 0.69 & 1.08 & \\
& $(0.52-0.97)$ & $(0.92-1.61)$ & $0.00004^{*}$ \\
TET3 & 0.19 & 0.39 & \\
& $(0.059-0.35)$ & $(0.34-0.65)$ & $0.00001^{*}$ \\
TDG & 0.47 & 0.23 & \\
\end{tabular}

(0.32-0.78)

$(0.2-0.3)$

Table 3. Expression of TET1, TET2, TET3 and TDG mRNA in the healthy controls and ALL patients $\left({ }^{*} \mathrm{p}<0.05\right)$.

\subsection{Protein expression analysis}

The protein expression of TET1 and TDG in the ALL group was significantly lower than that in the control group ( $p=0.007)$. In the case of the TET2 and TET3 proteins, we found no statistically significant differences (Table 4). The protein expression of TDG significantly decrease after treatment (median value 1.808 vs 1.413 respectively, $p=0.006$, Figure S1 in Supplementary Materials).

\begin{tabular}{cccc}
\hline Protein & $\begin{array}{c}\text { ALL } \\
\text { [protein level] }\end{array}$ & $\begin{array}{c}\text { Control } \\
\text { [protein level] }\end{array}$ & $\begin{array}{c}\text { ALL vs Control } \\
\text { P value }\end{array}$ \\
\hline TET1 & 1.19 & 1.6 & $0.007^{*}$ \\
& $(1.04-1.62)$ & $(1.4-1.92)$ & \\
TET2 & 1.3 & 1.48 & 0.52 \\
& $(1.08-1.91)$ & $(1.3-1.71)$ & \\
TET3 & 2.45 & 3.25 & 0.35 \\
& $(1.7-3.94)$ & $(2.36-3.61)$ & \\
\hline
\end{tabular}

Table 4. Expression of TET1, TET2 and TET3 protein in the lymphocytes of healthy controls and ALL patients $(* \mathrm{p}<0.05)$.

Interestingly, significant correlations between TETs ("producer") and TDG ("eraser") proteins were found (Figure S2-S5 in supplementary materials). A moderate positive correlations were observed between active DNA demethylation products and TET proteins in selected cell populations (Figure S6 in supplementary materials).

\subsection{Differences in the plasma concentrations of ascorbate}

Plasma concentrations of ascorbate in the ALL patients were lower than they were in the controls (median value 62.4 vs $97.8 \mu \mathrm{M}$ respectively, $\mathrm{p}=0.00027$, Figure 3).

\section{Discussion}

Acquiring an accurate measurement of 5-fdC, 5-cadC and 5-hmdU is challenging because not only are these modified bases in mammalian genomes expressed at very low levels but also the level of 5-hmdC is 
3-4 orders of magnitude higher than the levels of the other forms of modified DNA, which affects the detection and quantitation of the less common metabolites. To address this difficulty, we recently developed a rapid, highly sensitive and specific isotope-dilution method of automated two-dimensional ultra-performance liquid chromatography with tandem mass spectrometry (2D-UPLC-MS/MS) that is specifically tailored to analyze global levels of 5-mC, 5-hmC, 5-fC, 5-caC and 5-hmU simultaneously in cellular DNA and urine ${ }^{16,17}$. Importantly, many previous studies have used less reliable semiquantitative immunohistochemical methods to assess the levels of 5-hmC, 5-fC and 5-caC, and these results of these previous studies have lower reliability because of the low levels of modification in genomic DNA ${ }^{18}$.

Using the abovementioned methodology, we found a significant decrease in 5-hmdC in the leukocyte DNA of the ALL patients compared with the level in the healthy subjects. A similar decrease in this epigenetic modification has been found in many types of human malignancies ${ }^{18-21}$. One possible reason for the loss of 5-hmCyt is the decreased expression of TETs ${ }^{19,22}$. In agreement with this supposition, we found profound decreases in TET3 and TET2 mRNA expression in the leukocytes in the ALL patients. However, at the protein level, the only significant difference in the expression between the examined tissues was found for the case of TET1, which was significantly more abundant in the control group than it was in the patients. This difference may be linked with posttranslational context-dependent acetylation and deacetylation of TET lysine residues, which may enhance or reduce TET stability and activity ${ }^{23,24}$. Likely reason of the changes of TETs mRNA expression is methylation of $\mathrm{CpG}$ sites of promoter region which was found to correlate with gene expression changes in primary ALL cells ${ }^{13}$.

Another factor that influences the activity of TET proteins is vitamin C. Several previous studies demonstrated that ascorbate may enhance the generation of 5-hmCyt in cultured cells and under in vivo conditions ${ }^{25-27}$. Ascorbic acid (AA) enhances the activity of TETs, likely at the active site by reducing $\mathrm{Fe}^{3+}$ to $\mathrm{Fe}^{2+}$, which enables the donation of the electron needed to split an 0-0 into hydroxyl groups. Interestingly, the ALL patients analyzed in our study had significantly lower serum AA concentrations (Figure 3). Thus, a shortage of vitamin $\mathrm{C}$ may be another factor critical to the observed decrease in the 5$\mathrm{hmC}$ level in the leukocyte DNA of the patient group (in addition to decreased TET expression).

In ALL group, patients with ETV6/RUNX1 gene fusion (also known as TEL/AML1) have higher levels of analyzed modifications in urine and for 5-hmdC, 5-hmCyt, 5-fCyt and 5-hmUra the differences were significant (Table S3 in Supplementary Materials). Interestingly, a bias towards hypermethylation was found of the ETV6/RUNX1-positive pediatric ALL patients ${ }^{4,28}$. In the case of hyperdiploidy no differences between the subtypes have been found.

In patients treated with chemotherapy (methotrexate or epirubicin), the 8-oxodG level in leukocyte DNA and in urine was increased almost two fold compared to that of the same patient group before treatment. One of the components of the therapy drug cocktail is the anthracycline derivative epirubicin. The cytotoxicity of these drugs has been attributed to the inhibition of topoisomerase II and the intracellular production of free radicals. In our previous study, as in this study, significant increases in the amount of 
oxidatively modified DNA bases, including 8-oxoGua, compared to the control levels, was found in the lymphocytes of cancer patients treated with epirubicin ${ }^{29}$.

Urinary excretion rates of the majority of the DNA epigenetic modifications were significantly higher in the patient group than in the healthy subject group (Table 1). Recently, we and others found significant changes in urinary DNA epigenetic modifications only in 5-hmdC from patients with solid tumors and lymphoma patients ${ }^{17,30}$. This result may indicate the essential effects of epigenetic changes of DNA on the malignant transformation observed in pediatric ALL (see also below).

Importantly, a profound decrease in these modifications, to the level observed in the urine of the healthy subjects, was found after the completion of chemotherapy (Figure 2). The likely reason for this decrease is the massive death of cells with a high proliferative rate, namely, cancer cells. In addition, cancer cells have a higher turnover of epigenetic marks. Therefore, the abovementioned decrease may be linked with the recovery of the patients. Moreover, one of the components of the ALL treatment, methotrexate (MTX), acts by inhibiting dihydrofolate reductase, which is critical for the reduction in methyltetrahydrofolate and for methylation during DNA replication, which in turn directly reduces cell proliferation ${ }^{31}$. Furthermore, MTX is directly involved in the inhibition of DNA methylation in pediatric leukemia patients ${ }^{32}$.

Normal cell development requires the tight control of the DNA methylation pattern, which is involved in the regulation of gene expression. The methylation of cytosine, usually in $\mathrm{CpG}$ dinucleotides, is a key epigenetic modification that exerts a profound impact on gene repression, cellular identity and the maintenance of genome stability ${ }^{33}$. As mentioned in the Background, equally important for DNA methylation arrangement is the opposite reaction, namely, active DNA demethylation, which can result in activation of previously silenced genes. This process is directly linked with DNA repair and the removal of epigenetic modifications such as 5-fCyt and 5-caCyt (also 5-hmUra), which are replaced by unmodified cytosine. The removed bases (or deoxynucleosides) are released into the bloodstream and eventually appear in the urine ${ }^{34}$. Therefore, the most plausible source of the modifications excreted in the analyzed urine is the DNA repair process. Both 5-FCyt and 5-caCyt may inhibit DNA replication, which may result in genome instability ${ }^{35,36}$. Therefore, effective enzymatic systems are involved in the removal of modifications from DNA. Thus, TDG has robust excision activity toward 5-fCyt and 5-caCyt ${ }^{37-39}$. The activity of the abovementioned enzymes may contribute to the presence of modified bases in urine. Importantly, the expression of TDG mRNA was sharply increased in the ALL patients compared with controls (Table 3).

Another source of epigenetic marks excreted in urine, as analyzed in our study, may be the processive demethylation pathway, as proposed by Franchini et al. ${ }^{40,41}$. That is, 5-fCyt, 5-caCyt and 5-hmUra initiate processive DNA demethylation, which is primarily a single initiating event (such as a certain mismatch) that may trigger the processive demethylation of numerous 5-mCyts (and perhaps 5-hmCyts) on the same locus via the long-patch BER, DNA mismatch repair (MMR) or nucleotide excision repair (NER) pathways. Recent studies have demonstrated that 5-hmUra may trigger the removal of distant epigenetic 
modifications (5-mCyt and 5-hmCyt) through the MMR- and long-patch BER-dependent pathways ${ }^{42}$. These findings may explain the presence of 5-hmCyt and 5-mCyt deoxynucleosides in urine. Lesioncontaining oligomers in the NER/MMR/long-patch BER pathways may be the subject of intra/extracellular 5'-3' exonucleolytic digestion, which eventually results in the synthesis of 6- to 7nucleotide-long oligomers. However, the latter may be further degraded, and this poorly characterized post-excision processing may be eventually reflected in a modified deoxynucleotide yield ${ }^{43}$.

Interestingly, the urinary excretion rates found in this study for the control group/healthy children were similar to those we obtained for healthy adults, as described in our recently published study ${ }^{17}$. These findings seem to reflect basic processes that govern metabolic pathways critical for maintaining certain patterns of epigenetic DNA marks.

Several recently published studies have demonstrated that certain epigenetic alterations are essential for leukemic transformation ${ }^{13}$. The results of the aforementioned studies demonstrated the hypermethylation of GC islands in all subtypes of ALL. However, in these ALL cells, genome-wide hypomethylation was also frequently detected. Moreover, the ALL genomes were characterized by few somatic mutations, while the depletion of epigenetic modifications was simultaneously exacerbated in the majority of the pediatric ALL patients ${ }^{13}$. It is likely that profound changes in urinary excretion rates of epigenetically modified DNA in the ALL patients, in comparison with healthy children, as observed in this work, directly reflect the abovementioned dynamic changes in methylation signatures during disease development.

As explained above, a large body of evidence suggests that the level of 5-hmCyt in many human malignancies is substantially reduced ${ }^{18-21}$. Moreover, recently published data suggest that the decrease in the 5-hmCyt level may serve as a biomarker of early malignant transformation and can be used as a prognostic factor for patients with malignancies ${ }^{21,44}$. Consequently, the urinary modifications described herein may be used as potential risk and response markers. Owing to difficulties in obtaining specimens/tissues, such as bone morrow, especially from pediatric patients, the determination of epigenetic DNA modifications in human urine may serve as an attractive noninvasive diagnostic option. Furthermore, the noninvasiveness of the test constitutes a strong argument for its application to largescale basic research and clinical studies dealing with the role of active demethylation in carcinogenesis.

We found a highly significant increase in epigenetic DNA modifications in the urine of pediatric ALL patients compared with the level in healthy subjects. This increase likely reflects dynamic epigenetic changes in ALL pathogenesis. Importantly, after chemotherapy, urinary excretion rates of these modifications returned to the level characteristic of healthy children, a finding that may be linked with patient recovery. Our results suggest that the urinary excretion of epigenetically modified DNA may be a marker of pediatric ALL pathogenesis and a reliable marker of chemotherapy response. Since ETV6/RUNX1 fusion is favorable prognostic marker our results, which demonstrated direct link between the analyzed modifications in urine and ETV6/RUNX1 status, are a kind of support for the aforementioned statement. 


\section{Materials And Methods}

The subjects of the study were children $(n=55)$ with newly diagnosed acute lymphoblastic leukemia recruited in a hospital setting (Department of Pediatric Hematology and Oncology, Collegium Medicum, Nicolaus Copernicus University Torun, Jurasz University Hospital, Collegium Medicum, Bydgoszcz, Poland). Patients were treated according to ALL-IC-2009 or AIEOP-BFM ALL-2017 protocols. Peripheral blood and urine samples were collected before starting the treatment (point A), 33 days after the initiation of treatment (point B) and six months after the initiation of treatment (point $C$ - urine only). Samples obtained from healthy donors were used as controls $(n=21)$ with informed consent from their legal guardians. The clinical details of the patients are shown in Table 5. No active cytomegalovirus and Epstein-Barr virus infections were found in the studied groups. The standard panel of antibodies used to determine the immunophenotypic characterization included CD45, CD19, CD20, CD10, CD38 and CD58 for precursor-B-lineage and TdT, CD45, CD7, CD3, CD4, CD8, CD99, and cytoplasmic CD3 for T-lineage ALL 45 . All clinical investigations were conducted according to the principles of the Declaration of Helsinki. The protocol of the study was approved by the local Bioethics Committee at Collegium Medicum in Bydgoszcz, Nicolaus Copernicus University (KB 404/2016), and the guardians of all the patients provided written informed consent.

\begin{tabular}{|c|c|c|}
\hline & Patients, $\mathrm{n}=55$ & Control, $\mathrm{n}=21$ \\
\hline Age (years) & $5(1-18)$ & $8(2-17)$ \\
\hline \multicolumn{3}{|l|}{ Median (range) } \\
\hline Sex & $33 / 22$ & $13 / 8$ \\
\hline \multicolumn{3}{|l|}{ Male/Female (N) } \\
\hline Platelets $[/ \mu l]$ & $67000(125-457000)$ & \\
\hline \multicolumn{3}{|l|}{ Median (range) } \\
\hline WBC at diagnosis $[/ \mu \mathrm{l}]$ & $4840(33-5170000)$ & \\
\hline \multicolumn{3}{|l|}{ Median (range) } \\
\hline \multicolumn{3}{|l|}{ Median (range) } \\
\hline $\begin{array}{c}\text { \% Bone morrow (BM) blasts at diagnosis } \\
\text { Median (range) }\end{array}$ & $91(29-98)$ & \\
\hline \multicolumn{3}{|l|}{ Immunophenotype, n (\%) } \\
\hline T-ALL & $6(11)$ & \\
\hline B-ALL & 49 (89) & \\
\hline
\end{tabular}

Table 5. Baseline characteristics of the study groups.

\section{The determination of the epigenetic modifications and 8-oxodG levels in urine.}

Two-dimensional ultra-performance liquid chromatography with tandem mass spectrometry (2D UPLCMS/MS) was used for the epigenetic modification analysis of urine samples (with the exception of 5hmUra). Urine samples were spiked with a mixture of internal standards at a 4:1 volumetric ratio. The 2DUPLC-MS/MS system consists of a gradient pump and autosampler for one-dimensional chromatography, and a gradient pump and tandem quadrupole mass spectrometer with a UNISPRAY ion source was used for two-dimensional chromatography. Both systems were coupled with a column manager equipped with two programmable column heaters and two 2-position 6-port switching valves. 
The at-column dilution technique was used between the first and the second dimensions to improve the retention in the trap/transfer column. The sample molecules were then adsorbed to the packing material as very narrow bands that could be eluted with well-resolved, small-volume peaks. A diluting stream of water $(0.5 \mathrm{~mL} / \mathrm{min})$ was pumped with a Waters 515 isocratic pump and mixed with the first-dimension column effluent using a UPLC low-dead-volume tee valve. The following columns were used: CORTECS UPLC T3 Column $(1.6 \mu \mathrm{m}, 3 \mathrm{~mm} \times 150 \mathrm{~mm})$ with a CORTECS T3 VanGuard precolumn $(1.6 \mu \mathrm{m}, 2.1 \mathrm{~mm} \times$ $5 \mathrm{~mm})$ for the first dimension, a Waters ACQUITY UPLC CSH C18 $(1.7 \mu \mathrm{m}, 2.1 \mathrm{~mm} \times 100 \mathrm{~mm})$ for the second dimension, and a Waters XSelect CSH C18 column $(3.5 \mu \mathrm{m}, 3 \mathrm{~mm} \times 20 \mathrm{~mm})$ as the trap/transfer column. The chromatographic system was operated in heart-cutting mode, which means that selected portions of effluent from the first dimension were loaded onto the trap/transfer column by 6-port valve switching, which served as an "injector" for the second dimension of the chromatography system. Mass spectrometric detection was conducted with a Waters Xevo TQ-S tandem quadrupole mass spectrometer equipped with a UniSpray ionization source. The following common detection parameters were used: source temperature, $150^{\circ} \mathrm{C}$; nitrogen desolvation gas flow, $1000 \mathrm{~L} / \mathrm{h}$; nitrogen cone gas flow, $150 \mathrm{~L} / \mathrm{h}$; desolvation temperature, $500^{\circ} \mathrm{C}$; and nebulizer gas pressure, 7 bar. Collision-induced dissociation was obtained with argon (6.0 at $3 \times 10-6$ bar pressure) as a collision gas. The instrument response to all compounds was optimized by the infusion of $10 \mu \mathrm{M}$ genuine compounds dissolved in water (10 $\mu \mathrm{L} /$ minute) in the mobile phase A stream via the mass spectrometer fluidics system operating in the "mixed" mode using MassLynx 4.1 software IntelliStart feature. The quantitative and qualitative transition patterns and the specific settings of the detector are summarized in Table S5. The chromatographic system was operated with MassLynx 4.1 software from Waters. Quantitative analyses were performed using the TargetLynx application. All samples were analyzed with three to six technical replicates. Due to the low sensitivity of the method used, the level of 5-hmUra was determined by highperformance liquid chromatography for pre-purification followed by gas chromatography with isotope dilution mass spectrometric detection (LC/GC-MS), as previously described ${ }^{46}$.

\section{Isolation of DNA and the determination of the epigenetic modifications and 8-oxodG in DNA isolates}

Leukocytes were isolated from heparinized blood samples with Histopaque 1119 (Sigma) solution, according to the manufacturer's instructions, and stored at $-80^{\circ} \mathrm{C}$ until analysis. The analyses were performed using a method described earlier by Gackowski et al. with some modifications ${ }^{47}$. Briefly, a pellet of frozen cells was dispersed in ice-cold buffer $\mathrm{B}$ (Tris- $\mathrm{HCl}(10 \mathrm{mmol} / \mathrm{L}), \mathrm{Na}_{2}$ EDTA $(5 \mathrm{mmol} / \mathrm{L})$ and deferoxamine mesylate $(0.15 \mathrm{mmol} / \mathrm{L}), \mathrm{pH} 8.0)$. SDS solution was added (to a final concentration of $0.5 \%)$, and the mixture was gently mixed using a polypropylene Pasteur pipette. The samples were incubated at $37^{\circ} \mathrm{C}$ for 30 minutes. Proteinase $\mathrm{K}$ was added to a final concentration of $2.5 \mathrm{mg} / \mathrm{mL}$ and incubated at $37^{\circ} \mathrm{C}$ for $1.5 \mathrm{~h}$. The mixture was cooled to $4{ }^{\circ} \mathrm{C}$, transferred to a centrifuge tube with phenol:chloroform:isoamyl alcohol (25:24:1), and vortexed vigorously. After extraction, the aqueous phase was treated with a chloroform:isoamyl alcohol mixture (24:1). The supernatant was treated with two volumes of cold absolute ethanol to precipitate high molecular weight nucleic acids. The precipitate was removed with a plastic spatula, washed with 70\% (v/v) ethanol and dissolved in Milli-Q grade 
deionized water. The samples were mixed with $200 \mathrm{mM}$ ammonium acetate containing $0.2 \mathrm{mM} \mathrm{ZnCl}, \mathrm{pH}$ $4.6(1: 1 \mathrm{v} / \mathrm{v})$. Nuclease P1 $(1 \mathrm{U})$ and tetrahydrouridine $(10 \mu \mathrm{g} / \mathrm{sample})$ were added to the mixture and incubated at $37^{\circ} \mathrm{C}$ for $1 \mathrm{~h}$. Subsequently, $13 \mu \mathrm{L}$ and $15 \mu \mathrm{L}$ of $10 \%(\mathrm{v} / \mathrm{v}) \mathrm{NH}_{4} \mathrm{OH}$ (for the remaining nucleic acids and ultrafiltrate, respectively) and $1.3 \mathrm{U}$ of alkaline phosphatase were added to each sample and incubated for $1 \mathrm{~h}$ at $37^{\circ} \mathrm{C}$. Finally, all the hydrolysates were ultra-filtered prior to injection. The DNA hydrolysates were spiked with a mixture of internal standards at a volumetric ratio of $4: 1$ to a final concentration of $50 \mathrm{fmol} / \mu \mathrm{L}$ : $\left[\mathrm{D}_{3}\right]-5-\mathrm{hmdC},\left[{ }^{13} \mathrm{C}_{10},{ }^{15} \mathrm{~N}_{2}\right]-5-\mathrm{fdC},\left[{ }^{13} \mathrm{C}_{10},{ }^{15} \mathrm{~N}_{2}\right]-5$-cadC, and $\left[{ }^{13} \mathrm{C}_{10},{ }^{15} \mathrm{~N}_{2}\right]-5$ hmdU, $\left[{ }^{13} \mathrm{C},{ }^{15} \mathrm{~N}_{2}\right]-2$-deoxyuridine, and [ $\left.{ }^{15} \mathrm{~N}_{5}\right]-8-0 x o-7,8$-dihydro-2'-deoxyguanosine. Chromatographic separation was performed with a Waters ACQUITY 2D-UPLC system with a photodiode array detector for the first dimension of the 2D-chromatography (used for quantification of the unmodified deoxynucleosides and 5-mdC) and a Xevo TQ-S tandem quadrupole mass spectrometer (used for the second dimension of the 2D-chromatog nraphy to analyze the compounds from the first dimension in positive mode, namely, 5-hmdC and 8-oxodG, to assure better ionization at higher acetic acid concentrations). The at-column dilution technique was used between the first and second dimensions to improve the retention of the trap/transfer column. The following columns used: a Waters CORTECS T3 column $(150 \mathrm{~mm} \times 3 \mathrm{~mm}, 1.6 \mu \mathrm{m})$ with a precolumn for the first dimension, a Waters XSelect C18 CSH $(100 \mathrm{~mm} \times 2.1 \mathrm{~mm}, 1.7 \mu \mathrm{m})$ for the second dimension and a Waters XSelect C18 CSH $(20 \mathrm{~mm} \times 3 \mathrm{~mm}, 3.5$ $\mu \mathrm{m})$ column as a trap/transfer column. The chromatographic system was operated in heart-cutting mode, indicating that selected fractions of the effluent from the first dimension were loaded onto the trap/transfer column by 6-port valve switching, which served as the "injector" for the second dimension of the 2D-chromatography process. The flow rate for the first dimension was $0.5 \mathrm{~mL} /$ minute, and the injection volume was $2 \mu \mathrm{L}$. Separation was performed with a gradient elution for 10 minutes using a mobile phase of $0.05 \%$ acetate $(A)$ and acetonitrile (B) (0.7-5\% B for 5 minutes, column washing with $30 \%$ acetonitrile and re-equilibration with $99 \%$ A for 3.6 minutes). The flow rate for the second dimension was $0.3 \mathrm{~mL} /$ minute. The separation was performed with a gradient elution for 10 minutes using a mobile phase of $0.01 \%$ acetate $(A)$ and methanol (B) (1-50\% B for 4 minutes, isocratic flow of $50 \% B$ for 1.5 minutes, and re-equilibration with $99 \%$ A until the next injection). All samples were analyzed with three to five technical replicates, of which the technical mean was used for further calculation. Mass spectrometric detection was performed using a Waters Xevo TQ-S tandem quadrupole mass spectrometer equipped with an electrospray ionization source. Collision-induced dissociation was obtained using argon 6.0 at $3 \times 10^{-6}$ bar pressure as the collision gas. Transition patterns for all the analyzed compounds and the specific detector settings were determined using the MassLynx 4.1 IntelliStart feature set in quantitative mode to ensure the best signal-to-noise ratio and a resolution of 1 at MS1 and 0.75 at MS2.

\section{Determination of ascorbate level in blood plasma by UPLC-UV}

The analyses were performed using a method described earlier by Starczak et al. with some modifications $^{48}$. 


\section{Gene expression analysis}

RNA isolation was performed using the PAXgene blood RNA kit (Qiagen) following standard procedures. The concentration and purity of the RNA aliquots were verified spectrophotometrically with a NanoDrop 2000 spectrophotometer (Thermo Scientific). The $A_{260} / A_{280}$ ratio was used as an indicator of protein contamination, and the $\mathrm{A}_{260} / \mathrm{A}_{230}$ ratio was used as a measure of contamination with polysaccharides, phenol and/or chaotropic salts. The quality and integrity of the total RNA were assessed by visualization of the $28 \mathrm{~S} / 18 \mathrm{~S} / 5.8 S$ rRNA band pattern in a $1.2 \%$ agarose gel. Nondenaturing electrophoresis was carried out at $95 \mathrm{~V}$ for 20 minutes in TBE buffer (Tris, boric acid and EDTA). The gel was stained with ethidium bromide or SimplySafe and visualized using a G:BOX EF gel documentation system (Syngene).

The samples with RNA concentrations greater than $50 \mathrm{ng} / \mu \mathrm{L}$ were qualified for further analysis. A total of

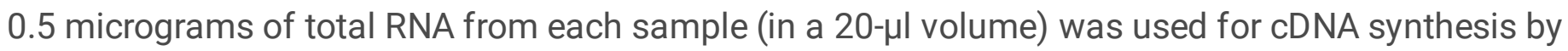
reverse transcription with a high-capacity cDNA reverse transcription kit (Applied Biosystems, catalog no. 43-688-14) according to the manufacturer's instructions. The reaction was carried out with a Mastercycler nexus gradient thermocycler (Eppendorf). To exclude contamination with genomic DNA, reverse transcriptase reaction also included a negative control. cDNA was either immediately used for qPCR or stored at $-20^{\circ} \mathrm{C}$.

Gene transcripts were analyzed by relative quantitative RT-PCR (RT-qPCR) with relevant primers and short hydrolysis probes substituted with locked nucleic acids from the Universal Probe Library (UPL, Roche Table 6). The probes were labeled with fluorescein (FAM) at the 5'-end and with a dark quencher dye at the 3 '-end. The expression of target genes was normalized against four selected reference genes: HMBS (GenelD: 3145), TBP (GenelD: 6908), ACTB (GenelD: 60) or G6PD (GenelD: 2539) using a UPL ready

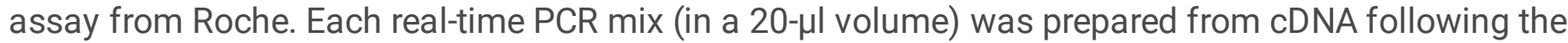
standard procedures for the LightCycler 480 Probe Master Mix (Roche), as provided with the reagent set. The reactions were carried out on 96-well plates. In addition to the proper samples, each plate also included a no-template control and no-RT control. Quantitative real-time PCR was carried out on a LightCycler $480 \mathrm{II}$ using the following cycling parameters: $10 \mathrm{~s}$ at $95^{\circ} \mathrm{C}$, followed by 45 repeats of $10 \mathrm{~s}$ each at $95^{\circ} \mathrm{C}, 30 \mathrm{~s}$ at $58^{\circ} \mathrm{C}$, and finally, $1 \mathrm{~s}$ at $72^{\circ} \mathrm{C}$ in the acquisition mode (with the parameters of wavelength excitation and detection set to $465 \mathrm{~nm}$ and $510 \mathrm{~nm}$, respectively). The reaction for each gene was standardized against a standard curve to estimate amplification efficiency. The standardization procedure included the preparation of 10 -fold serial dilutions with a controlled relative amount of targeted template. The efficiency of amplification was assessed based on the slope of the standard curve. Standard dilutions were amplified in separate wells but within the same run. The analyses were performed using a method described earlier by Dziaman et al. with some modifications ${ }^{49}$. 


\begin{tabular}{cccc}
\hline Gene & Forward primer sequence & Reverse primer sequence & $\begin{array}{c}\text { UPL } \\
\text { (Roche) }\end{array}$ \\
\hline TET1 & 5'-tctgttgttgtgcctctgga-3' & 5'-gcctttaaaactttgggcttc-3' & $\# 57$ \\
TET2 & 5'-gcctttgctcctgttgagtt-3' & 5'-acaaggctgccctctagttg-3' & $\# 38$ \\
\hline TET3 & 5'-cactccggagaagatcaagc-3' & 5'-ggacaatccaccttcagag-3' & $\# 1$ \\
\hline TDG & 5'-GAATGGAAGCGGAGAACG-3' & 5'-TTGCTGTTCATTCACAACTGC-3' & $\# 41$ \\
\hline
\end{tabular}

Table 6. Primers and short hydrolysis probes used for the target gene mRNA expression analysis.

\section{Protein expression analysis}

Because of the lack of commercially available anti-TETs (Tet1, Tet2, Tet3) antibodies recommended for direct flow cytometry method, it was decided to use an indirect staining consisting of 2 steps involving labeling with unconjugated primary and compatible fluorochrome-conjugated secondary antibodies. Both type of antibodies were titrated.

This process enables the determination of the antibody amount and concentration, resulting in the highest possible signal from the positive population and the lowest signal from the negative population. Nonspecific antibody-binding was eliminated by optimizing the amount and concentration of the antibody ${ }^{50}$.

The following antibodies were used: 1) rabbit polyclonal anti-Tet1 antibody (Cat. No. ab121587), Abcam; 2) goat polyclonal anti-Tet2 antibody (Cat. No. ab99432), Abcam; 3) rabbit polyclonal anti-Tet3 antibody (Cat. No. ab139311).

The secondary antibodies were selected based on the primary antibodies and conjugated with a fluorochrome: 1) donkey F(ab')2 anti-rabbit IgG H\&L (Alexa Fluor® 488) (Cat. No. ab181346, Abcam); 2) rabbit $F\left(a b^{\prime}\right) 2$ anti-goat IgG H\&L (Alexa Fluor ${ }^{\circledR}$ 647) (Cat. No. ab169347, Abcam).

For titration, the following primary antibody amounts were used: $0.5 \mu \mathrm{l}, 0.6 \mu \mathrm{l}, 0.8 \mu \mathrm{l}, 1 \mu \mathrm{l}$, and $1.2 \mu \mathrm{l}$. The secondary antibody dilutions were 1:400 $(\mu \mathrm{l}), 1: 1000(\mu \mathrm{l}), 1: 2000(\mu \mathrm{l})$, and 1:4000 $(\mu \mathrm{l})$. In the first step, the secondary antibody was titrated, and a dilution of 1:1000 was selected. Then, the primary antibodies were titrated, and $1 \mu \mathrm{l}$ was chosen. Perm/Wash Buffer from BD Cytofix/Cytoperm ${ }^{\mathrm{TM}}$ (BD Biosciences) was used for the dilutions.

Blood specimens were collected by venipuncture into tubes containing tripotassium EDTA and the cellular surface antigen-stabilizing agent TransFix. Before the staining procedure was performed, the cells were counted using a LUNA-II automated cell counter. This number was important for ensuring the correct amount of antibodies was added. For the purposes of our experiment, a volume of blood containing 100 000 cells was added to a $5-\mathrm{ml}$ round-bottom tube and suspended in PBS to obtain a final volume of 100 $\mu \mathrm{l}$.

Fluorochrome-labeled monoclonal antibodies: PE-anti-human CD56/CD16, PerCP-anti-human CD14, APCH7-anti-human CD19, BV42-anti-human CD45, V500-anti-human CD3, and PE-Cy7-anti-human CD34 (BD 
Biosciences, Franklin Laker, New Jersey, USA) were added to each of the tubes according to the scheme shown in Table 7 with an amount recommended by the manufacturer.

\begin{tabular}{c|c}
\hline Number of tubeS & MONOCLONAL ANTIBODIES \\
\hline 1. & CD45BV421/CD34PE-Cy7 \\
2. & CD45BV421/CD56+CD16PE/CD14PerCP/CD19APC-H7/CD3V500/CD34PE-Cy7 \\
3. & CD45BV421/CD56+CD16PE/CD14PerCP/CD19APC-H7/CD3V500/CD34PE-Cy7 \\
4. & CD45BV421/CD56+CD16PE/CD14PerCP/CD19APC-H7/CD3V500/CD34PE-Cy7 \\
\hline
\end{tabular}

Table 7. Panel of surface labelling markers.

The samples were incubated at room temperature in the dark for 30 minutes. Then, the erythrocytes were lysed with $2 \mathrm{ml}$ of FACS lysing solution (BD Biosciences) and incubated for 15 minutes under the same conditions as the previous incubation. After two washes with PBS and centrifugation at $500 \times \mathrm{g}$ for 5 minutes, the cell pellets were resuspended in $500 \mu \mathrm{l}$ of BD Cytofix/Cytoperm ${ }^{\text {TM }}$ solution (BD Biosciences) to fix and permeabilize the cells. The cells were incubated in the dark for 20 minutes at room temperature. Then, the cells were centrifuged at $500 \mathrm{xg}$ for 5 minutes again. Cell pellets were resuspended in freshly prepared Perm/Wash Buffer and incubated for 10 minutes in the dark. Then, the cells were centrifuged, the supernatant was removed, and the pellets were treated by primary antibodies to the scheme shown in Table 8.

\begin{tabular}{c|cc}
\hline $\begin{array}{c}\text { Number of } \\
\text { tubeS }\end{array}$ & PRIMARY ANTIBODIES & SECONDARY ANTIBODIES \\
\hline 1. & Perm wash buffer & Perm wash buffer \\
2. & Perm wash buffer & $\begin{array}{c}\text { Donkey anti-rabbit Alexa Fluor 488/Rabbit } \\
\text { anti-goat Alexa Fluor 647 }\end{array}$ \\
\hline 3. & Rabbit polyclonal anti-human TET-1 & Donkey anti-rabbit Alexa Fluor 488 \\
\cline { 2 - 3 } 4. & $\begin{array}{c}\text { Rabbit polyclonal anti-human TET-3/goat } \\
\text { polyclonal anti human TET-2/ }\end{array}$ & $\begin{array}{c}\text { Donkey anti-rabbit Alexa Fluor 488/rabbit anti- } \\
\text { goat Alexa Fluor 647 }\end{array}$ \\
\hline
\end{tabular}

Table 8. Panel of intracellular labelling.

The secondary antibody solution of donkey anti-rabbit Alexa Fluor 488 or rabbit anti-goat Alexa Fluor 647 (Abcam) was prepared and $100 \mu \mathrm{L}$ of the 1000 -fold diluted antibody was added to the samples. One hundred microliters of Perm wash buffer was added to tube no. 1. Sample no. 2, which included only surface and secondary antibodies, was a control for the investigated proteins. The tubes were incubated at room temperature for 30 minutes in the dark. Then, $1 \mathrm{~mL}$ of Perm wash buffer was added per tube and incubated for 10 minutes at room temperature in the dark. Next, the samples were centrifuged $(500 \times \mathrm{g}$ for 5 minutes), and the supernatant was removed by pipette. The cell pellets were resuspended in $250 \mu \mathrm{L}$ of PBS and then analyzed using a three-laser BD FACS Canto II flow cytometer (BD Biosciences) ${ }^{51}$. The instrument was set up using a BD Cytometer Setup and Tracking beads. Data on 30,000 cells were acquired with BD FACSDiva software and analyzed with FlowJo 7.5.5 software (Tree Star Inc., Ashland, Oregon, USA). 
Cell doublets were rejected by setting the gate for single cells in the plot linear forward-scatter area (FSCA) vs. linear forward-scatter height (FSC-H). The leukocytes (granulocytes, monocytes and lymphocytes, and blasts) were gated on the basis of their morphological features, as measured by linear side-scatter area (SSC-A) and CD45 antigen expression (logarithmic CD45 Brilliant Violet 421-H). For each population, the fluorescence was detected separately and plotted in histogram mode: counts vs. logarithmic Alexa Fluor 488-H and counts vs. logarithmic Alexa Fluor 647-H. The geometric mean of the fluorescence intensity (GMFI) was calculated for the total cell population in each channel. The expression of the investigated proteins was calculated as the geometric mean fluorescence intensity in the test sample as a fold change over the negative control (tube no. 2) (ratio= GMFI test tube/GMFI control tube).

\section{Statistical analysis}

The results are presented as median values, interquartile ranges and non-outlier ranges. Statistical analyses were carried out with Statistica 13.1 PL software [Dell Inc. (2016). Dell Statistica, version 13. software.dell.com.].Variables with a normal distribution were analyzed as "raw" data, while variables with non-normal distributions were subjected to Box-Cox transformation prior to statistical analyses based on parametric tests. The results were considered statistically significant at $p$ values less than 0.05 .

\section{Abbreviations}

ALL: acute lymphoblastic leukemia; TET: ten-eleven translocation; 5-mdC: 5-methyl-2'-deoxycytidine; 5mCyt: 5-methylcytosine; 5-hmCyt: 5-hydroxymethylcytosine; 5-fCyt: 5-formylcytosine; 5-caCyt: 5carboxycytosine; 5-hmUra: 5-hydroxymethyluracil; 2D-UPLC-MS/MS: two-dimensional ultra-performance liquid chromatography with tandem mass spectrometry; 8-oxodG: 8-oxo-7,8-dihydro-2'-deoxyguanosine; 5mdC: 5-methyl-2'-deoxycytidine; 5-hmdC: 5-(hydroxymethyl)-2'-deoxycytidine; 5-fdC: 5-formyl-2'deoxycytidine; 5-cadC: 5-carboxy-2'-deoxycytidine; 5-hmdU: 5-(hydroxymethyl)-2'-deoxyuridine; MLL: mixed lineage leukemia; AML: acute myeloid leukemia; LC/GC-MS: liquid chromatography followed by gas chromatography with isotope dilution mass spectrometric detection; UPLC-UV: ultra-performance liquid chromatography coupled with UV detection; BER: base excision repair; MMR: DNA mismatch repair; NER: nucleotide excision repair; RT-qPCR: quantitative real-time PCR

\section{Declarations}

\section{Data availability}

The datasets obtained in the current study are available from the corresponding author on reasonable request.

\section{Acknowledgments}

This work was supported by the Polish National Science Center [Grant No. 2015/19/B/NZ5/02208]. RR, DG, AS, MS, ASG, EZ, MM, TD, ASz (Anna Szpila), KL, JG, JS, MG, AL, MF, and RO are a members of 
University Center of Excellence "Towards Personalized Medicine" operating under Excellence Initiative Research University. RO dedicates this paper to the memory of Prof. Barbara Tudek.

\section{Author Contributions}

$\mathrm{RR}, \mathrm{DG}, \mathrm{JS}$ and RO designed the study, analyzed data, wrote the manuscript and coordinated the research. RR, DG, AS (Aleksandra Skalska), MS, ASG, EZ, MM, TD, AS (Anna Szpila), KL. JG, JS, MG, AL, LG, MF, EO, AK performed the laboratory work for this study. AS (Aleksandra Skalska), MS, EZ, MM, AS (Anna Szpila), KL, JS, MG, AL, LG, EO, AK contributed to sample collection. ASG, TD, JG and MF contributed to data interpretation. All authors read and approved the final manuscript.

\section{Competing interests}

The authors declare no conflict of interest.

\section{References}

1 Pui, C. H. \& Evans, W. E. Acute lymphoblastic leukemia. N Engl J Med 339, 605-615, doi:10.1056/NEJM199808273390907 (1998).

2 Mrozek, K., Harper, D. P. \& Aplan, P. D. Cytogenetics and molecular genetics of acute lymphoblastic leukemia. Hematol Oncol Clin North Am 23, 991-1010, v, doi:10.1016/j.hoc.2009.07.001 (2009).

3 Roman-Gomez, J. et al. Promoter hypermethylation and global hypomethylation are independent epigenetic events in lymphoid leukemogenesis with opposing effects on clinical outcome. Leukemia 20, 1445-1448, doi:10.1038/sj.leu.2404257 (2006).

4 Wong, N. C. et al. A distinct DNA methylation signature defines pediatric pre-B cell acute lymphoblastic leukemia. Epigenetics 7, 535-541, doi:10.4161/epi.20193 (2012).

5 Nordlund, J., Milani, L., Lundmark, A., Lonnerholm, G. \& Syvanen, A. C. DNA methylation analysis of bone marrow cells at diagnosis of acute lymphoblastic leukemia and at remission. PLoS One 7, e34513, doi:10.1371/journal.pone.0034513 (2012).

6 Kohli, R. M. \& Zhang, Y. TET enzymes, TDG and the dynamics of DNA demethylation. Nature 502, 472-479, doi:10.1038/nature12750 (2013).

7 Song, C. X. \& He, C. Potential functional roles of DNA demethylation intermediates. Trends Biochem Sci 38, 480-484, doi:10.1016/j.tibs.2013.07.003 (2013).

8 Bhutani, N., Burns, D. M. \& Blau, H. M. DNA Demethylation Dynamics. Cell 146, 866-872, doi:10.1016/j.cell.2011.08.042 (2011). 
Cooke, M. S. et al. DNA repair is responsible for the presence of oxidatively damaged DNA lesions in urine. Mutation Research-Fundamental and Molecular Mechanisms of Mutagenesis 574, 58-66, doi:10.1016/j.mrfmmm.2005.01.022 (2005).

10 Pronier, E. \& Delhommeau, F. Role of TET2 mutations in myeloproliferative neoplasms. Curr Hematol Malig Rep 7, 57-64, doi:10.1007/s11899-011-0108-8 (2012).

11 Imbesi, S. et al. Oxidative stress in oncohematologic diseases: an update. Expert Rev Hemato/6, 317-325, doi:10.1586/ehm.13.21 (2013).

12 Yang, Y. et al. Determinants of urinary 8-hydroxy-2'-deoxyguanosine in Chinese children with acute leukemia. Environ Toxicol 24, 446-452, doi:10.1002/tox.20447 (2009).

13 Nordlund, J. \& Syvanen, A. C. Epigenetics in pediatric acute lymphoblastic leukemia. Semin Cancer Bio/ 51, 129-138, doi:10.1016/j.semcancer.2017.09.001 (2018).

14 Tasian, S. K. \& Hunger, S. P. Genomic characterization of paediatric acute lymphoblastic leukaemia: an opportunity for precision medicine therapeutics. Br J Haematol 176, 867-882, doi:10.1111/bjh.14474 (2017).

15 Shurtleff, S. A. et al. TEL/AML1 fusion resulting from a cryptic $t(12 ; 21)$ is the most common genetic lesion in pediatric ALL and defines a subgroup of patients with an excellent prognosis. Leukemia 9, 1985-1989 (1995).

16 Gackowski, D. et al. Accurate, Direct, and High-Throughput Analyses of a Broad Spectrum of Endogenously Generated DNA Base Modifications with Isotope-Dilution Two-Dimensional Ultraperformance Liquid Chromatography with Tandem Mass Spectrometry: Possible Clinical Implication. Anal Chem 88, 12128-12136, doi:10.1021/acs.analchem.6b02900 (2016).

17 Rozalski, R., Gackowski, D., Siomek-Gorecka, A., Banaszkiewicz, Z. \& Olinski, R. Urinary Measurement of Epigenetic DNA Modifications: A Non-Invasive Assessment of the Whole-Body Epigenetic Status in Healthy Subjects and Colorectal Cancer Patients. Chemistryopen 5, 550-553, doi:10.1002/open.201600103 (2016).

18 Yang, H. et al. Tumor development is associated with decrease of TET gene expression and 5methylcytosine hydroxylation. Oncogene 32, 663-669, doi:10.1038/onc.2012.67 (2013).

$19 \mathrm{Li}, \mathrm{W}$. \& Liu, M. Distribution of 5-hydroxymethylcytosine in different human tissues. J Nucleic Acids 2011, 870726, doi:10.4061/2011/870726 (2011).

20 Jin, S. G. et al. 5-Hydroxymethylcytosine Is Strongly Depleted in Human Cancers but Its Levels Do Not Correlate with IDH1 Mutations. Cancer Research 71, 7360-7365, doi:10.1158/0008-5472.Can-112023 (2011). 
21 Lian, C. G. et al. Loss of 5-Hydroxymethylcytosine Is an Epigenetic Hallmark of Melanoma. Cell 150, 1135-1146, doi:10.1016/j.cell.2012.07.033 (2012).

22 Cimmino, L., Abdel-Wahab, O., Levine, R. L. \& Aifantis, I. TET family proteins and their role in stem cell differentiation and transformation. Cell Stem Cel/ 9, 193-204, doi:10.1016/j.stem.2011.08.007 (2011).

23 Sun, J. et al. SIRT1 Activation Disrupts Maintenance of Myelodysplastic Syndrome Stem and Progenitor Cells by Restoring TET2 Function. Cell Stem Cell 23, 355-369 e359, doi:10.1016/j.stem.2018.07.018 (2018).

24 Zhang, Q. et al. Tet2 is required to resolve inflammation by recruiting Hdac2 to specifically repress IL-6. Nature 525, 389-393, doi:10.1038/nature15252 (2015).

25 Yin, R. C. et al. Ascorbic Acid Enhances Tet-Mediated 5-Methylcytosine Oxidation and Promotes DNA Demethylation in Mammals. J Am Chem Soc 135, 10396-10403, doi:10.1021/ja4028346 (2013).

26 Minor, E. A., Court, B. L., Young, J. I. \& Wang, G. Ascorbate induces ten-eleven translocation (Tet) methylcytosine dioxygenase-mediated generation of 5-hydroxymethylcytosine. J Biol Chem 288, 1366913674, doi:10.1074/jbc.C113.464800 (2013).

27 Blaschke, K. et al. Vitamin C induces Tet-dependent DNA demethylation and a blastocyst-like state in ES cells. Nature 500, 222-226, doi:10.1038/nature12362 (2013).

28 Figueroa, M. E. et al. Integrated genetic and epigenetic analysis of childhood acute lymphoblastic leukemia. J Clin Invest 123, 3099-3111, doi:10.1172/JCl66203 (2013).

29 Olinski, R., Jaruga, P., Foksinski, M., Bialkowski, K. \& Tujakowski, J. Epirubicin-induced oxidative DNA damage and evidence for its repair in lymphocytes of cancer patients who are undergoing chemotherapy. Mol Pharmacol 52, 882-885, doi:10.1124/mol.52.5.882 (1997).

30 Zhang, Y. F., Qi, C. B., Yuan, B. F. \& Feng, Y. Q. Determination of cytidine modifications in human urine by liquid chromatography - Mass spectrometry analysis. Anal Chim Acta 1081, 103-111, doi:10.1016/j.aca.2019.07.002 (2019).

31 Wang, Y. C. \& Chiang, E. P. Low-dose methotrexate inhibits methionine S-adenosyltransferase in vitro and in vivo. Mol Med 18, 423-432, doi:10.2119/molmed.2011.00048 (2012).

32 Vezmar, S., Schusseler, P., Becker, A., Bode, U. \& Jaehde, U. Methotrexate-associated alterations of the folate and methyl-transfer pathway in the CSF of ALL patients with and without symptoms of neurotoxicity. Pediatr Blood Cancer 52, 26-32, doi:10.1002/pbc.21827 (2009).

33 Feng, S., Jacobsen, S. E. \& Reik, W. Epigenetic reprogramming in plant and animal development. Science 330, 622-627, doi:10.1126/science.1190614 (2010). 
in urine. Mutation Research-Fundamental and Molecular Mechanisms of Mutagenesis 574, 58-66, doi:10.1016/j.mrfmmm.2005.01.022 (2005).

35 Schiesser, S. et al. Mechanism and Stem-Cell Activity of 5-Carboxycytosine Decarboxylation Determined by Isotope Tracing. Angew Chem Int Edit 51, 6516-6520, doi:10.1002/anie.201202583 (2012).

36 Munzel, M. et al. Improved Synthesis and Mutagenicity of Oligonucleotides Containing 5Hydroxymethylcytosine, 5-Formylcytosine and 5-Carboxylcytosine. Chem-Eur J 17, 13782-13788, doi:10.1002/chem.201102782 (2011).

37 He, Y. F. et al. Tet-Mediated Formation of 5-Carboxylcytosine and Its Excision by TDG in Mammalian DNA. Science 333, 1303-1307, doi:10.1126/science.1210944 (2011).

38 Maiti, A. \& Drohat, A. C. Thymine DNA Glycosylase Can Rapidly Excise 5-Formylcytosine and 5Carboxylcytosine POTENTIAL IMPLICATIONS FOR ACTIVE DEMETHYLATION OF CpG SITES. J Biol Chem 286, 35334-35338, doi:10.1074/jbc.C111.284620 (2011).

39 Olinski, R., Starczak, M. \& Gackowski, D. Enigmatic 5-hydroxymethyluracil: Oxidatively modified base, epigenetic mark or both? Mutat Res-Rev Mutat 767, 59-66, doi:10.1016/j.mrrev.2016.02.001 (2016).

40 Franchini, D. M. et al. Processive DNA Demethylation via DNA Deaminase-Induced Lesion Resolution. Plos One 9, doi:ARTN e9775410.1371/journal.pone.0097754 (2014).

41 Franchini, P., Fruciano, C., Frickey, T., Jones, J. C. \& Meyer, A. The Gut Microbial Community of Midas Cichlid Fish in Repeatedly Evolved Limnetic-Benthic Species Pairs. Plos One 9, doi:ARTN e9502710.1371/journal.pone.0095027 (2014).

42 Grin, I. \& Ishchenko, A. A. An interplay of the base excision repair and mismatch repair pathways in active DNA demethylation. Nucleic Acids Res 44, 3713-3727, doi:10.1093/nar/gkw059 (2016).

43 Olinski, R. et al. Urinary measurement of 8-oxodG, 8-oxoGua, and 5HMUra: A noninvasive assessment of oxidative damage to DNA. Antioxidants \& Redox Signaling 8, 1011-1019, doi:10.1089/ars.2006.8.1011 (2006).

44 Chen, M. L. et al. Quantification of 5-Methylcytosine and 5-Hydroxymethylcytosine in Genomic DNA from Hepatocellular Carcinoma Tissues by Capillary Hydrophilic-Interaction Liquid Chromatography/Quadrupole TOF Mass Spectrometry. Clinical Chemistry 59, 824-832, doi:10.1373/clinchem.2012.193938 (2013).

45 Dworzak, M. N. et al. Standardization of Flow Cytometric Minimal Residual Disease Evaluation in Acute Lymphoblastic Leukemia: Multicentric Assessment Is Feasible. Cytom Part B-Clin Cy 74b, 331340, doi:10.1002/cyto.b.20430 (2008). 

biomarkers in patients with colorectal cancer. Biomarkers 20, 287-291, doi:10.3109/1354750X.2015.1068860 (2015).

47 Gackowski, D. et al. Accurate, Direct, and High-Throughput Analyses of a Broad Spectrum of Endogenously Generated DNA Base Modifications with Isotope-Dilution Two-Dimensional Ultraperformance Liquid Chromatography with Tandem Mass Spectrometry: Possible Clinical Implication. Anal Chem 88, 12128-12136, doi:10.1021/acs.analchem.6b02900 (2016).

48 Starczak, M. et al. In vivo evidence of ascorbate involvement in the generation of epigenetic DNA modifications in leukocytes from patients with colorectal carcinoma, benign adenoma and inflammatory bowel disease. J Trans/ Med 16, doi:ARTN 20410.1186/s12967-018-1581-9 (2018).

49 Dziaman, T. et al. Characteristic profiles of DNA epigenetic modifications in colon cancer and its predisposing conditions-benign adenomas and inflammatory bowel disease. Clin Epigenetics 10, doi:ARTN 72 10.1186/s13148-018-0505-0 (2018).

$50 \quad$ Uhlen, M. et al. Towards a knowledge-based Human Protein Atlas. Nat Biotechno/ 28, 12481250, doi:10.1038/nbt1210-1248 (2010).

51 Dagur, P. K. \& McCoy, J. P., Jr. Collection, Storage, and Preparation of Human Blood Cells. Curr Protoc Cytom 73, 51 1-5 116, doi:10.1002/0471142956.cy0501s73 (2015).

\section{Figures}

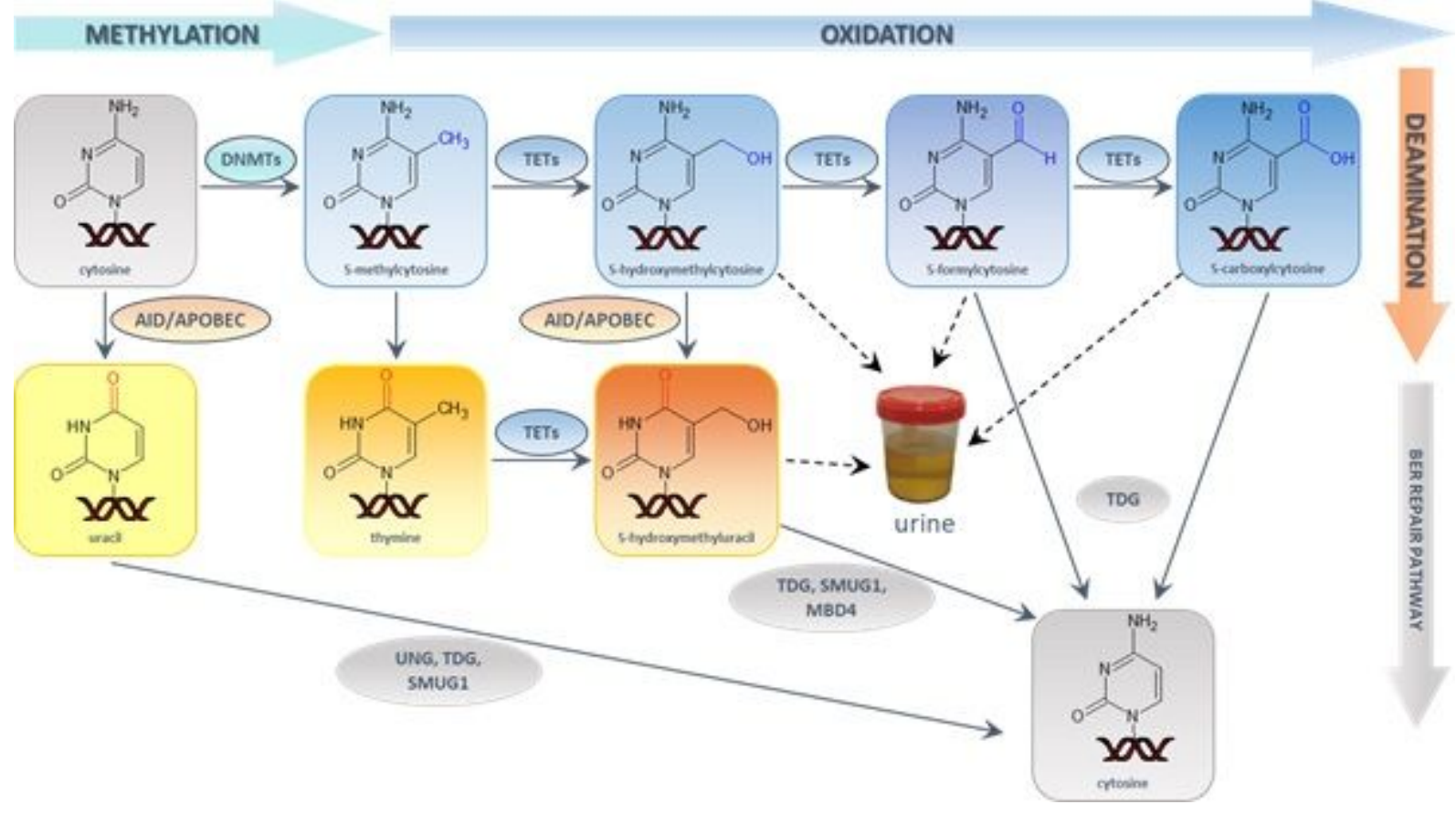

Figure 1 
Cytosine methylation and active demethylation pathway. (DNMT - DNA methyltransferase, TETs - Ten Eleven Translocation enzymes, AID - activation-induced cytosine deaminase, TDG - thymine DNA glycosylase, BER - base excision repair). Reprinted from Biochimica et Biophysica Acta - Reviews on Cancer, 1869 (1), Olinski R, Gackowski D, Cooke M. Endogenously generated DNA nucleobase modifications source, and significance as possible biomarkers of malignant transformation risk, and role in anticancer therapy , 29-41 (2018) with permission from Elsevier.

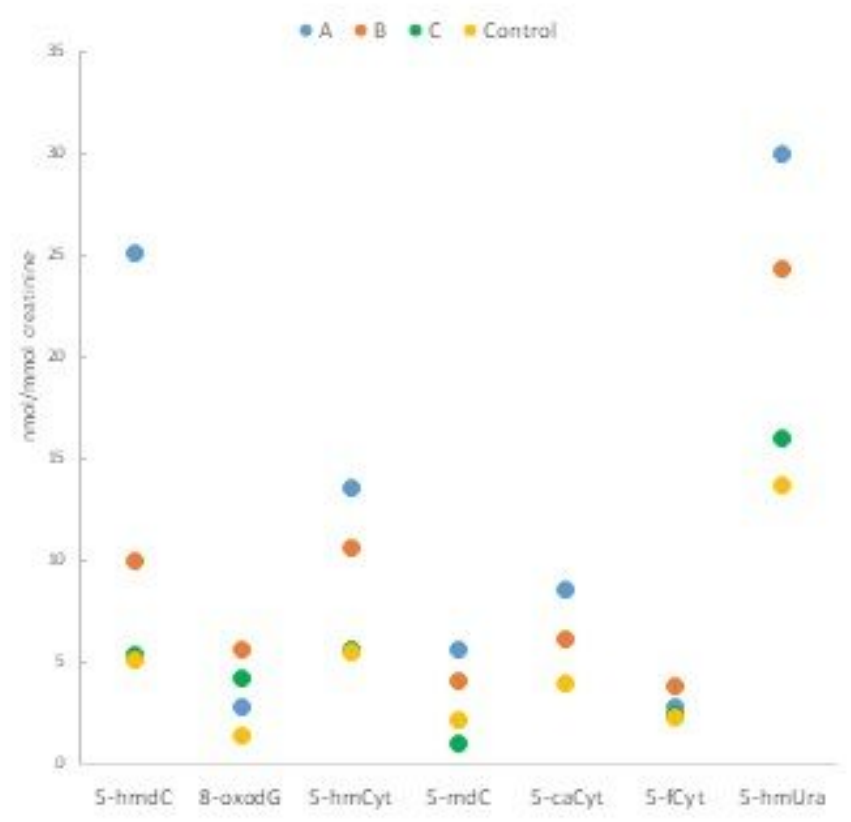

\section{Figure 2}

Urinary levels of DNA damage markers and active demethylation products of 5-methylcytosine in the healthy controls and ALL patients before therapy (A), 30 days after treatment (B) and six months after treatment $(\mathrm{C})$. The results are presented as median values. 


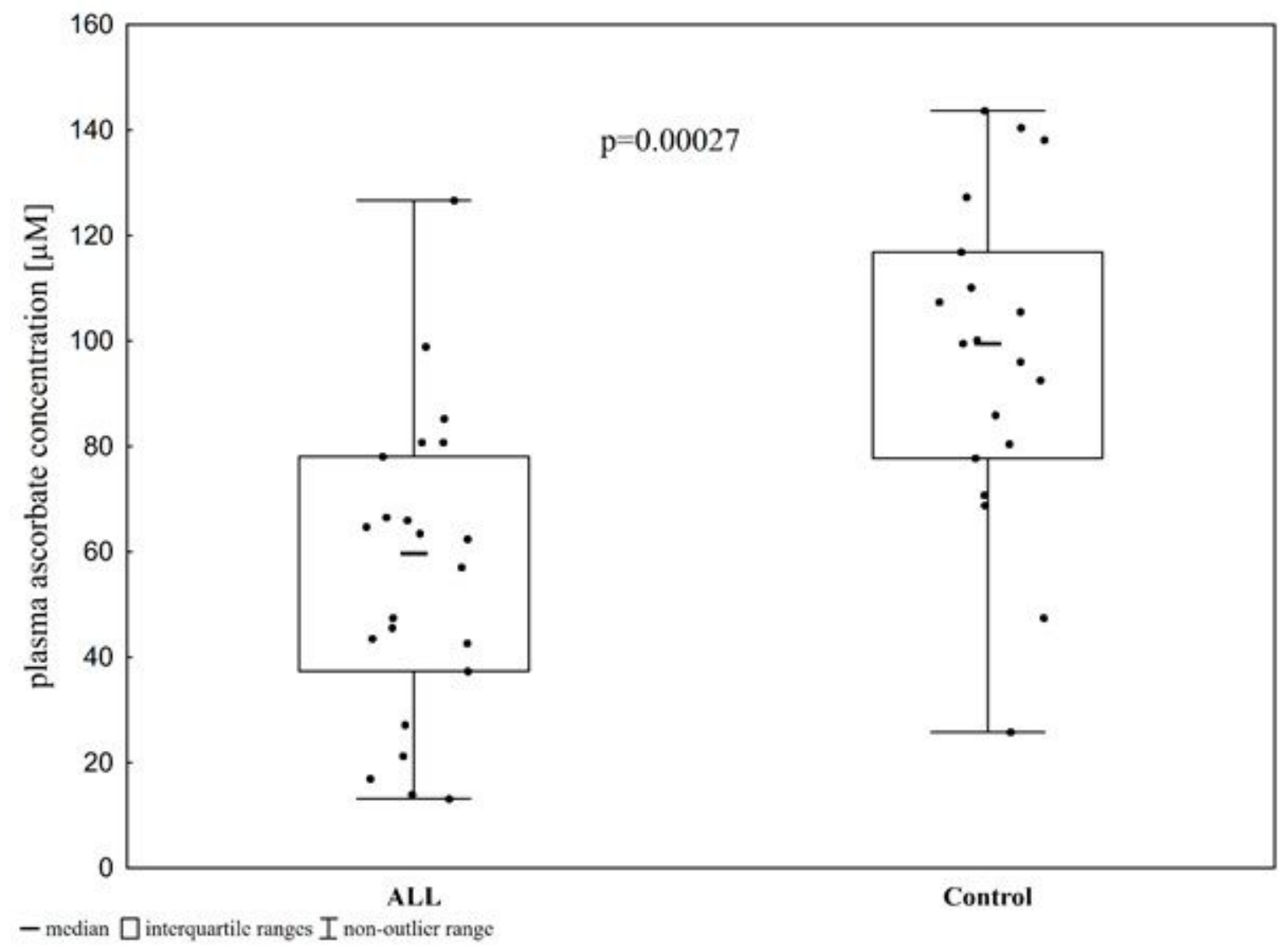

Figure 3

Ascorbic acid in blood plasma.

\section{Supplementary Files}

This is a list of supplementary files associated with this preprint. Click to download.

- SupplementaryMaterial.pdf 